\title{
CONSERVATIVE DYNAMICAL SYSTEMS INVOLVING STRONG FORCES
}

\author{
BY \\ WILLIAM B. GORDON
}

\begin{abstract}
We consider conservative dynamical systems associated with potentials $V$ which have singularities at a set $S: V(x) \rightarrow-\infty$ as $x \rightarrow S$. It is shown that various "action" integrals satisfy Condition $C$ of Palais and Smale provided that the potential satisfy a certain strong force (SF) condition. Hence, e.g., we establish the existence in SF systems of periodic trajectories which wind around $S$ and have arbitrary given topological (homotopy) type and which have arbitrary given period, and also periodic trajectories which make arbitrarily tight loops around $S$. Similar results are also obtained concerning the existence of trajectories which wind around $S$ and join two given points. The SF condition is shown to be closely related to the completeness (in the riemannian sense) of certain Jacobi metrics associated with the potential $V$, and this fact permits the use of the standard results of riemannian geometry in the analysis of SF systems. The SF condition excludes the gravitational case, and the action integrals do not satisfy the Palais-Smale condition in the gravitational case. The Jacobi metrics associated with gravitational potentials are not complete. For SF systems there exist trajectories which join two given points and make arbitrarily tight loops around $S$, and this is not the case in the gravitational two body problem. On the other hand, for SF systems any smooth family of $\lambda$-periodic trajectories ( $\lambda$ fixed) is bounded away from $S$, and this also is not the case for gravitational systems. Thus the definition of the SF condition is "well motivated", and leads to the disclosure of certain differences between the behavior of SF systems and gravitational (and other weak force) systems.
\end{abstract}

1. Introduction and statement of main existence theorems.

1A. Introduction. In this paper we shall describe an attempt to obtain information about $n$-body systems and other kinds of conservative dynamical systems through the application of standard variational and geometrical techniques to the standard variational principles of classical mechanics.

We shall be considering systems of the general type

$$
\ddot{x}+\nabla V(x)=0
$$

where $x=\left(x^{1}, \cdots, x^{N}\right)$ denotes a general point of $\mathrm{R}^{N}$ and $V$ is a real-valued 
function of $\mathbf{R}^{N}$ with gradient $\nabla V$. Throughout this paper it will always be assumed that $V$ is of class $C^{2}$ everywhere on $\mathbf{R}^{N}$ except at a closed nonempty set $S$ at which $V$ has infinitely deep wells, i.e., it will be assumed that $V(x) \rightarrow$ $-\infty$ as $x \rightarrow \mathbf{S}$.

DEFINITION. The system (1.1) will be said to satisfy the strong-force (SF) condition if and only if

There exists a neighborhood $\mathbf{N}$ of $\mathbf{S}$ and a $C^{2}$ function $U$ on $\mathbf{N}-\mathbf{S}$ such that

(i) $U(x) \rightarrow-\infty$ as $x \rightarrow \mathrm{S}$, (ii) $-V(x) \geqslant|\nabla U(x)|^{2}$ for all $x$ in $\mathbf{N}-\mathbf{S}$.

EXAMPLE 1.1. Let $S$ consist of a single point, the origin. Recall that a potential $V$ gives rise to a force $-\nabla V$, so that the inverşe-square potential $V(x)=-|x|^{-2}$ gives rise to the inverse-cube force $\nabla V=-|x|^{-4} x$ which is easily shown to be strong, since in this case we can set $U(x)=\log |x|$.

EXAMPLE 1.2. The inverse-square force corresponding to the gravitational potential $V(x)=-|x|^{-1}$ is weak (not strong). For let $U$ be a function which satisfies (ii) in condition (SF). Then $U(x)$ can behave no worse than $|x|^{1 / 2}$ in a neighborhood of the origin, contradicting requirement (i).

REMARK 1.1. It should be emphasized that condition (SF) is a condition on the local (near-field) behavior of a potential in a neighborhood of its singularities. Thus our results do not at all apply to, say, magnetic dipoles whose inverse-cube character is a far-field property.

REMARK 1.2. Many of our results will apply to the more general case of conservative dynamical systems defined on riemannian manifolds. In this case, the first term of the left-hand side of (1.1) must be replaced with the appropriate covariant derivative. Note also that condition (SF) is coordinate free and is therefore immediately applicable to riemannian manifolds.

Our definition of the SF condition has the following motivation: Let $\Sigma$ be some appropriate Sobolev space which consists of either $\lambda$-periodic cycles (= smooth closed paths) in $\mathbf{R}^{N}-\mathbf{S}$, or of paths which join two given points in $\mathbf{R}^{N}-\mathbf{S}$ with a given time of transit. For any element $f$ of $\Sigma$ whose euclidean coordinate representation is given by $x=x(t)$ set

$$
A(f)=\int\left\{1 / 2|\dot{x}(t)|^{2}-V(x(t))\right\} d t .
$$

According to Hamilton's principle the extremals of the functional $A$ are solutions to (1.1). Hence, one would like to exhibit solutions to (1.1) as extremals, i.e., as critical points to some functional (such as $A$ ), and then proceed to locate 
(and compute) such critical points by means of standard methods in the calculus of variations-specifically, the following:

(i) Methods using lower semicontinuity properties of the functionals.

(ii) Direct methods, such as the Ritz method.

(iii) Gradient methods, such as the method of steepest descent.

(iv) The topological theories of Morse and Lusternick-Schnirelmann. Technically, this means that we have to establish that the functionals satisfy Condition C of Palais and Smale.

(v) Techniques from riemannian geometry. In this case solutions to (1.1) with given total energy are represented as geodesics with respect to a certain "Jacobi" metric.

The main theme of this paper is to show that this rather simple-minded approach works provided that the SF condition is satisfied.

It is disappointing that the gravitational case is excluded by the SF condition (cf. Example 1.2). However, the failure of these methods in the gravitational case is both systematic and interesting, i.e., the SF condition is "well motivated", and our analysis based on its use will serve to disclose some of the special character of gravitational and other weak-force systems.

We should also mention that our analysis is based on the use of functionals, such as (1.2), which are integrals whose integrands are quadratic in $\dot{x}$, and it may be that there are functionals of a different character for which the methods enumerated above will work in the weak-force case.

1B. Notation and preliminary definitions. For any fixed number $\lambda>0, \Sigma^{1}$ $=\Sigma^{\mathbf{l}}\left(\lambda ; \mathbf{R}^{N}-\mathbf{S}\right)$ will denote the Sobolev space of all absolutely continuous $\lambda$. periodic cycles which lie in $\mathbf{R}^{N}-\mathbf{S}$ and have $L^{2}$ derivatives defined almost everywhere. For any pair of (not necessarily distinct) points $p, q$ in $\mathbf{R}^{N}-\mathbf{S}$ and real number $T>0$,

$$
\Omega^{1}=\Omega^{1}\left(T ; p, q ; \mathbf{R}^{N}-\mathrm{S}\right)
$$

will denote the Sobolev space of all absolutely continuous paths $f=f(t)$ which lie in $\mathbf{R}^{N}-\mathrm{S}$, have $L^{2}$ derivatives almost everywhere, and which join $p$ to $q$ in time $T: f(0)=p$ and $f(T)=q$. These spaces will be discussed in more detail in $\S \S 5$ and 7 , but at this point we should mention that $\Sigma^{1}\left(\lambda ; \mathbf{R}^{N}-S\right)$ is an open submanifold of the Hilbert space $\Sigma^{1}\left(\lambda ; R^{N}\right)$, and that an admissible norm for $\Sigma^{1}\left(\lambda ; \mathbf{R}^{N}\right)$ is given by $\|f\|_{1}^{2}=\|f\|_{0}^{2}+\|D f\|_{0}^{2}$ where $\|\cdot\|_{0}$ denotes the ordinary $L^{2}$ norm and $D=d / d t$. By the Sobolev imbedding theorems, weak convergence in $\|\cdot\|_{1}$ implies uniform convergence, i.e., the weak $\Sigma^{1}$ topology is stronger than the $C^{0}$ topology. (Similar remarks apply of course to the spaces $\Omega^{1}$.) 
For any path or cycle $f$ in $\Omega^{1}$ or $\Sigma^{1}$ with euclidean representation $x=x(t)$ we define the following functionals:

$$
\begin{aligned}
& J(f)=1 / 2 \int|\dot{x}(t)|^{2} d t=1 / 2\|D f\|_{0}^{2}=1 / 2\|f\|_{1}^{2}-1 / 2\|f\|_{0}^{2}, \\
& E(f)=\int V(x(t)) d t .
\end{aligned}
$$

Hence, from (1.2) we can write

$$
A=J-E .
$$

Our main results consist of a number of theorems which assert that any cycle or path joining two given points can be continuously deformed in $\mathbf{R}^{N}-\mathbf{S}$ into a solution of (1.1), provided that the path or cycle winds around $S$ in a sufficiently complicated way. The definitions below make this notion precise.

Definition 1.1. A smooth path $f$ joining two given points $p, q$ in $\mathbf{R}^{N}-\mathbf{S}$ will be said to be simple if $p=q$ and $f$ is homotopic in $\mathbf{R}^{N}-\mathbf{S}$ to a point, or if $p \neq q$ and $f$ belongs to the homotopy class of paths in $\mathbf{R}^{N}-\mathbf{S}$ which contains the line segment $\overline{p q}$. This homotopy class will be understood to be empty if $\overline{p q}$ intersects $\mathbf{S}$, in which case every path in $\mathbf{R}^{N}-\mathbf{S}$ which joins $p$ to $q$ is nonsimple.

Definition 1.2. A smooth cycle $f$ in $\mathbf{R}^{N}-\mathbf{S}$ will be said to be tied to $\mathbf{S}$ if $f$ cannot be continuously moved off to infinity without either crossing $\mathbf{S}$ or having its arc length become infinite. More precisely, a cycle $f$ is tied to $\mathbf{S}$ if for every $c>0$ there exists a (possibly empty) compact subset $K_{c}$ of $\mathbf{R}^{N}$ which contains every smooth cycle which is homotopic to $f$ in $\mathbf{R}^{N}-\mathbf{S}$ and has arc length $\leqslant c$.

EXAmple 1.3. Let $\mathbf{R}^{N}=\mathbf{R}^{2}$ and let $S$ be a discrete set of points. Then a cycle $f$ is tied to $\mathbf{S}$ if and only if $f$ is not homotopic (in $\mathbf{R}^{2}-\mathbf{S}$ ) to a point, but this is not a general fact, as the next example will show.

EXAmple 1.4. Let $\mathbf{R}^{N}=\mathbf{R}^{3}$, and let $\mathrm{S}$ be the union of three straight lines which intersect at the origin. Then a cycle $f$ is tied to $\mathbf{S}$ if and only if $f$ winds around at least two of the lines. A cycle which winds around only one of the lines is not tied to $\mathbf{S}$. Although this example has no great physical interest, the next example will show that it provides a good pictorial representation of the geometry involved in planar $n$-body problems.

EXAMPLE 15. Let there be three masses $m_{i}$ with position coordinates $\left(x_{i}, y_{i}\right)$ constrained to move in the plane $\mathbf{R}^{2}$, so that the configuration space of the system is $\mathbf{R}^{6}$. We suppose that each mass attracts every mass along the lines joining them. Reduce the dimension of the configuration space from six to four by fixing the centroid at the origin. The singularities of the system correspond to collisions of the masses, and these are defined by the three coincidence relations 
$P_{i}:\left\{x_{j}=x_{k}\right.$ and $\left.y_{j}=y_{k}\right\}$, where the triple $\{i, j, k\}$ varies over the cyclic permutations of $\{1,2,3\}$. Hence the configuration space of the reduced system with the singularities removed is $\mathbf{R}^{4}-\mathbf{S}$ where $\mathbf{S}$ is the union of the three 2-planes $P_{i}$. Any pair of these planes intersect at precisely one point (the origin). A cycle $f$ is tied to $S$ if and only if it winds around at least two of the planes. The property of winding around the plane $P_{i}$ can be defined by the nonvanishing of the period $\int_{f} \omega_{i}$ where $\omega_{i}$ is the closed but nonexact differential 1-form which corresponds to an infinitesimal rotation around the plane $\boldsymbol{P}_{\boldsymbol{i}}$. (Similar remarks apply to planar $n$-body systems, $n>3$.)

1C. Statement of main existence theorems. The components of the spaces $\Omega^{1}$ and $\Sigma^{1}$ correspond to homotopy classes of paths or cycles, and in the first theorem presented below we shall exhibit solutions to (1.1) as paths or cycles at which $A$ attains minimum values on components. In the second theorem solutions are obtained as paths or cycles at which $J$ is minimized subject to the constraint $E=$ constant.

THEOREM 1.1. Assume condition (SF), and in addition that $V$ is bounded above, say, $V<0$. Then

(a) For every $\lambda>0$, every homotopy class of cycles in $\Sigma^{1}\left(\lambda ; \mathbf{R}^{N}-\mathbf{S}\right)$ which consists of cycles tied to $\mathbf{S}$ contains a cycle $f^{*}$ at which A attains a minimum value (for that homotopy class). Each such cycle $f^{*}$ is a $\lambda$-periodic solution to (1.1).

(b) For every $T>0$, and for every pair of (not necessarily distinct) points $p, q$ in $\mathbf{R}^{N}-\mathbf{S}$, every homotopy class of paths in $\Omega^{1}\left(T ; p, q ; \mathbf{R}^{N}-\mathbf{S}\right)$ contains a path $f^{*}$ at which A attains a minimum value, and each such path $f^{*}$ is a solution to (1.1) which joins $p$ to $q$ with time of transit $T$.

REMARK 1.3. The paths in part (b) of this theorem are not required to be nonsimple.

Notation. For each real number $c$ let $\Sigma_{c}^{1}=\Sigma^{1} \cap E^{-1}\{c\}$ and $\Omega_{c}^{1}=\Omega^{1}$ $\cap E^{-1}\{c\}$. The sets $\Sigma_{c}^{1}$ and $\Omega_{c}^{1}$ may be empty for certain values of $c$, e.g., if $V<0$ and $c \geqslant 0$.

The next theorem asserts that $J$ can be minimized subject to the constraint $E=c$ on each homotopy class of, say, $2 \pi$-periodic cycles tied to $\mathbf{S}$, i.e., $J$ attains minimum values on each component $\Gamma$ of $\Sigma_{c}^{1}=\Sigma_{c}^{1}\left(2 \pi ; \mathbf{R}^{N}-S\right)$. (Similar remarks apply to $\Omega_{c}^{1}$.) Each cycle $f^{*}$ providing such a minimum is a $2 \pi$-periodic solution to

$$
\ddot{x}(t)+\mu \nabla V(x(t))=0
$$

where $\mu$ is a Lagrange multiplier which is necessarily nonzero if $\Gamma$ consists of cycles which are not homotopic to a point. If $\mu>0$, then (1.5) provides a 
solution to (1.1) after the transformation of parameter $t \rightarrow t \sqrt{ } \mu$. If $\mu<0$, the transformation of parameter $t \rightarrow t \sqrt{ }-\mu$ provides a solution to $(1.1)^{*}$ where

$$
\ddot{x}-\nabla V(x)=0 .
$$

Hence minimizing $J \mid \Sigma_{c}^{1}$ provides periodic solutions to either (1.1) or (1.1)*. The periods of these solutions cannot be specified because the values of the Lagrange multipliers $\mu$ cannot be determined a priori. (Similar remarks apply to $J \mid \Omega_{c}^{1}$; in this case the requirement that the component consist of nonsimple paths will insure that $\mu \neq 0$.)

The device of exhibiting solutions to (1.1) as extremals for $J \mid \Sigma_{c}^{1}$ is due to M. S. Berger [2] , [3] , [4] , and has been used by him and the author [7] to obtain information about the existence of periodic solutions to dynamical systems with convex potentials.

From the Schwarz inequality we have

$$
\operatorname{arc~length}(f)=\int_{0}^{2 \pi}|\dot{x}(t)| d t \leqslant(4 \pi J(f))^{1 / 2}
$$

with equality if and only if the parameter $t$ of $f: x=x(t)$ is proportional to arc length. Hence, once it is established that $J$ is minimized on each component of $\Sigma_{c}^{1}$ (for which $\Sigma_{c}^{1} \neq \varnothing$ ), it will readily follow that there exist solutions which wind around $\mathbf{S}$ and have arbitrarily small arc length subject to the requirement that the solutions have a prescribed topological type; or, to put the matter another way, we obtain solutions which wind around and come arbitrarily close to $\mathbf{S}$.

We would like to be able to exclude (1.1)* as a possibility. Now the set of singularities $\mathbf{S}$ can be described as a set of attractors for the system (1.1), and as a set of repulsors for system (1.1)*, and in most physically realistic situations one can indeed exclude the possibility of having solutions which wind around and come arbitrarily close to a system of repulsors S. However, there do exist examples which show that this is not the general case, i.e., there do exist systems of repulsors S for which (1.1)* has periodic solutions tied to S. See Remark 1.5 and Example 1.6 following the statement of the theorem.

THEOREM 1.2. Assuming condition SF, then:

(a) Every nonempty component (homotopy class) of $\Sigma_{c}^{1}=\Sigma_{c}^{1}\left(2 \pi ; \mathbf{R}^{N}-\mathbf{S}\right)$ which consists of cycles which are tied to $\mathbf{S}$ and not homotopic to a point contains a cycle $f^{*}$ at which $J$ attains a minimum value, and each such cycle $f^{*}$ is a periodic solution to (1.5) where the Lagrange multiplier $\mu$ is nonzero. Hence, given any $\epsilon>0$, any cycle $f$ which is tied to $\mathbf{S}$ and not homotopic to a point can be continuously deformed (in $\mathbf{R}^{N}-\mathbf{S}$ ) into a periodic solution (with unspecified period) of either (1.1) or (1.1)* which at some points comes to within an $\epsilon$ distance of $\mathbf{S}$. 
(b) Similarly, every homotopy class of nonsimple paths (in $\mathbf{R}^{N}-\mathbf{S}$ ) joining two given points $p, q$ in $\mathbf{R}^{N}-\mathbf{S}$ contains an infinite number of solutions to either (1.1) or (1.1)* which join $p$ to $q$ and cut arbitrarily small neighborhoods of $\mathbf{S}$.

REMARK 1.4. Suppose $\mathbf{R}^{N}=\mathbf{R}^{2}$ and that $\mathbf{S}$ consists of a single attractor located at the origin. Let $p, q$ be two distinct points in $\mathbf{R}^{N}-\mathbf{S}$, and suppose that the SF condition is satisfied. Then Theorem 1.2(b) provides for the existence of an infinite number of solutions to (1.1) which join $p$ to $q$ and make arbitrarily tight loops around the origin. Such solutions do not exist in the gravitational case described in Example 1.2.

REMARK 1.5. Versions of these theorems (with errors) were announced in [8] and [9]. In particular, Condition [A] in [9] is the SF condition, and Condition [B] of the same paper was alleged to exclude $(1.1)^{*}$ as a possibility. The following example provides a counterexample to this.

EXAMPLE 1.6. Consider the case of two negative electrical charges which are fixed in space at positions $p$ and $q$ respectively. Then a third negative charge can be made to oscillate back and forth along a segment of the line $\overline{p q}$, and can be made to come arbitrarily close to the repulsors $p, q$. In this case two "infinitely high" potential wells join together to form a convex well with a critical point (of relative minimum) between $p$ and $q$. The periodic trajectories thus obtained are not tied to the set of singularities; however, if we modify this system by surrounding the charges with a sphere of negative electricity, we obtain a new system for which (1.1)* cannot be excluded as a possibility for cycles which are tied to and come arbitrarily close to $\mathbf{S}$.

The next theorem is based on the use of Jacobi's principle, according to which trajectories with given total energy can be represented as geodesics with respect to a certain riemannian metric (the "Jacobi" metric).

ThEOREM 1.3. Assume the SF condition, and in addition that $V<0$. Let $p$ and $q$ be two distinct points in $\mathbf{R}^{N}-\mathbf{S}$, and let $\Gamma$ be any homotopy class of paths in $\mathbf{R}^{N}-\mathbf{S}$ which join $p$ to $q$. Then for every $h>0, \Gamma$ contains a trajectory to (1.1) with total energy $h$. If $p=q$, the same assertion holds, provided that $\Gamma$ does not consist of paths which are homotopic (in $\mathbf{R}^{N}-\mathbf{S )}$ to a point.

The proof will be given in $\$ 4 \mathrm{D}$. It turns out that condition (SF) is almost, but not quite, equivalent to the assertion that the Jacobi metrics associated with (1.1) and values of $h>0$ are complete (in the riemannian sense), and in $\S 4$ we shall discuss a slightly stronger version of the SF condition which does in fact insure the completeness of these metrics and a fortiori the assertions in the theorem. 
A discussion of a geometrical method which is valid for the case $h<0$, and also for weak-force systems, is given in $\$ 4 \mathrm{E}$.

1D. Three lemmas. The first lemma (G) formalizes the role that the SF condition will play in the proofs, and lies at the heart of our discussion. It will be proved in $\S 2$.

Lemma G (Main geometrical lemma). Assume condition SF. Then any family $\Gamma$ of homotopic cycles in $\Sigma^{1}\left(\lambda ; \mathbf{R}^{N}-\mathbf{S}\right)$ which are tied to $\mathbf{S}$, and on which $|E|$ and $J$ are bounded, is bounded away from $\mathbf{S}$. That is, if there exist constants $c_{1}, c_{2}$ such that $|E(f)|<c_{1}$ and $J(f)<c_{2}$ for all $f$ in $\Gamma$, then there exists a number $\delta>0$ such that no cycle $f$ in $\Gamma$ intersects the $\delta$-neighborhood of S. Similarly, any family of (not necessarily simple) paths in $\Omega^{1}\left(T ; p, q ; \mathbf{R}^{N}-\mathbf{S}\right)$ on which $|E|$ and $J$ are bounded is bounded away from $\mathbf{S}$.

The two "analytical" lemmas $A_{1}$ and $A_{2}$ are stated for reasons of explicitness. The first lemma $A_{1}$ is standard, but the second lemma $A_{2}$ is not quite so standard and is concerned with some regularity results which are needed for the application of the theory of Sobolev spaces to variational problems. Proofs are given in $\$ 5 B$ and $\$ 7 C$.

These lemmas are used in the proof of Theorem 1 and, strictly speaking, there should be another set of lemmas which are applicable to the case of Theorem 2. However, these lemmas are really concerned with the calculation of the gradients of the functionals $E$ and $J$ on the spaces $\Sigma^{1}$ and $\Omega^{1}$, and their application to the proof of Theorem 2 will follow from the extension of the method of Lagrange multipliers to functions defined on infinite dimensional spaces.

Lemma $A_{1}$. The critical points of $A \mid \Sigma^{1}\left(\lambda ; R^{N}-S\right)$ are precisely the $\lambda$ periodic solutions to (1.1).

LEMMA $A_{2}$. The critical points of $A \mid \Omega^{1}\left(T ; p, q ; \mathbf{R}^{N}-\mathrm{S}\right)$ are precisely the solutions to (1.1) which join $p$ to $q$ in time $T$.

\section{Proof of Lemma G.}

2A. We first prove the lemma for cycles. Let

$$
\mathbf{G}=\left\{(x, z) \in \mathrm{R}^{N+1}: x \in \mathrm{R}^{N}-\mathrm{S}, z=U(x)\right\}
$$

be the graph of the comparison function $U=U(x)$ referred to in the definition of the SF condition. For any cycle $f: x=x(t)$ belonging to $\Sigma^{1}\left(\lambda ; R^{N}-S\right)$ let

$$
\widetilde{f}:\{x=x(t), z=U(x(t))\}
$$


be the lifting of $f$ to $\mathbf{G}$, and let $\sigma=\sigma(\widetilde{f})$ be the arc length of $\widetilde{f}$. Then using elementary inequalities, one obtains

$$
\dot{\sigma}^{2}=|\dot{x}|^{2}+|\dot{z}|^{2}=|\dot{x}|^{2}+\langle\dot{x}, \nabla U\rangle^{2} \leqslant|\dot{x}|^{2}+|\dot{x}|^{2}|\nabla U|^{2},
$$

so that $\dot{\sigma} \leqslant|\dot{x}|+|\dot{x}||\nabla U|$, and

$$
\sigma=\int \dot{\sigma} d t \leqslant\left[\lambda \int|\dot{x}|^{2} d t\right]^{1 / 2}+\left[\int|\dot{x}|^{2} d t\right]^{1 / 2}\left[\int|\nabla U|^{2} d t\right]^{1 / 2}
$$

Now, since we are only concerned with the behavior of $V$ in a neighborhood of $\mathrm{S}$, there is no loss of generality in assuming that $V \leqslant 0$ and $-V \geqslant|\nabla U|^{2}$. Hence, from (2.1), we write

$$
\sigma(\tilde{f}) \leqslant(2 \lambda J(f))^{1 / 2}+(2 J(f) \cdot|E(f)|)^{1 / 2} .
$$

We are given a family $\Gamma$ of cycles $f$ for which $J(f) \leqslant c_{1}$ and $E(f) \leqslant$ $c_{2}$ for all $f$ belonging to $\Gamma$. Hence

$$
\sigma(\tilde{f}) \leqslant c_{3}, \text { for all } f \in \Gamma .
$$

For reasons of clarity we shall first proceed to prove the lemma for the special case $\mathbf{R}^{N}=\mathbf{R}^{2}$ and $\mathbf{S}=\{p, q\}, p \neq q$, and then generalize. The proof for this special case can be further reduced to the special cases when the family $\Gamma$ consists of a sequence of cycles $f_{n}$ all of which wind around one or both of the singularities.

Case (i). All the $f_{n}$ wind around $p$ only. Suppose the $f_{n}$ are not bounded away from $p$. Then, for every $\epsilon>0$, the $\epsilon$-neighborhood of $p$ intersects an infinite number of cycles $f_{n}$. By passing to a subsequence, we can suppose that

$$
\inf \left\{\left|f_{n}(t)-p\right|: 0 \leqslant t \leqslant \lambda\right\} \rightarrow 0 \text { as } n \rightarrow \infty .
$$

Now, from a geometric or pictorial point of view, this last statement is equivalent to saying that portions of the lifted cycles $\widetilde{f}_{n}$ fall infinitely far down the well of $\mathbf{G}$ at $p$ as $n \rightarrow \infty$.

Suppose there exists a $\delta>0$ such that portions of an infinite number of the $f_{n}$ fall outside the $\delta$-neighborhood of $\mathbf{S}$. Then the variation of $U\left(f_{n}(t)\right)$ would become infinite as $n \rightarrow \infty$, but $\sigma\left(\widetilde{f_{n}}\right)$ is greater than the variation of $U\left(f_{n}(t)\right.$ ) (which geometrically is the length of the projection of $\tilde{f}_{n}$ onto the $z$ axis), and this contradicts (2.3).

On the other hand, suppose there exists no such $\delta$. Then the $f_{n}$ collapse to the point $p$, which implies that $E\left(f_{n}\right) \rightarrow-\infty$, contradicting the hypotheses.

Case (ii). All the $f_{n}$ wind around both $p$ and $q$. In this case the proof is even quicker, since $\sigma\left(\widetilde{f}_{n}\right)>d(p, q)+$ variation in $U\left(f_{n}(t)\right)$. 
2B. We now proceed to prove the lemma for general $\mathbf{R}^{N}$ and $\mathbf{S}$. Again, we can suppose that $\Gamma$ is a sequence of cycles $f_{n}=f_{n}(t)$ which, upon being reparametrized by arc length, converge uniformly to a cycle $f^{*}$. If the $f_{n}$ are not bounded away from $S$, then $f^{*}$ will be attached to $S$ at one or more points. If $f^{*}$ is attached to only one point of $\mathbf{S}$ the argument of Case (i) above applies. If $f^{*}$ is attached to more than one point, the argument of Case (ii) applies.

This completes the proof of the lemma for cycles. The proof of the lemma for the path spaces $\Omega^{1}\left(T ; p, q ; \mathbf{R}^{N}-\mathbf{S}\right)$ is similar to the argument in Case (ii).

3. Proofs of Theorems 1.1 and $\mathbf{1 . 2}$ based on lower semicontinuity properties.

3A. We recall some properties of lower semicontinuous (1.s.c.) functions.

Let $F: X \rightarrow \mathbf{R}$ be a real valued function on a topological space $X$. Then $F$ is 1.s.c. if and only if $F^{-1}(-\infty, c]$ is closed for every real number $c$, in which case $F$ is bounded below and attains its infimum on every compact subset of $X$. If $X$ is Hausdorff, then compact sets are necessarily closed, and we have the following proposition.

Proposition 3.1. Suppose $F: X \rightarrow \mathbf{R}$ is a real valued function on the Hausdorff space $X$ which has the property that

$$
F^{-1}(-\infty, b] \text { is compact for every real } b \text {. }
$$

Then $F$ is l.s.c., bounded below, and attains its minimum value on $X$.

Proof. Take a value of $b$ such that $F^{-1}(-\infty, b]$ is nonempty. Then the infimum of $F$ on $F^{-1}(-\infty, b]$ is the infimum of $F$ on $X$.

In the sequel $X$ will be a subset of a hilbert space with the (induced) weak topology. We recall that a subset of a hilbert space is weakly compact if and only if it is weak-closed and bounded.

3B. We shall now assume the hypotheses of Theorem 1.1 and prove that the functional $A$ attains minimum values. (The remainder of the theorem, which asserts that such minimizing cycles or paths are solutions to (1.1), is provided by Lemmas $A_{1}$ and $A_{2}$ whose proofs are given in $\$ 5 B$ and $\S 7 C$.)

Let $X$ be a component of $\Sigma^{1}\left(\lambda ; R^{N}-S\right)$ which consists of cycles tied to $S$, and endow $X$ with the weak topology it inherits from $\Sigma^{1}\left(\lambda ; \mathbf{R}^{N}\right)$. We wish to apply Proposition 3.1 with $F=A$, i.e., we have to show that $X \cap$ $A^{-1}(-\infty, b]$ is a bounded and weak-closed subset of $\Sigma^{1}\left(\lambda ; \mathbf{R}^{N}\right)$.

Since $J=A+E$ and $V<0$, we have $E<0$ and therefore

$$
\begin{aligned}
& J \leqslant b \text { on } A^{-1}(-\infty, b]=A^{-1}[0, b], \\
& |E|=-E=A-J \leqslant b \text { on } A^{-1}[0, b] .
\end{aligned}
$$


From (3.2) it follows that the elements of $X$ are bounded in arc length and from Lemma $\mathbf{G}$ it follows that the elements of $X$ are bounded away from $\mathbf{S}$; and since the elements of $X$ are tied to $S$, it follows that they are bounded in $C^{0}$ norm, and hence in the $L^{2}$ norm $\|\cdot\|_{0}$ (defined in $\S 1 \mathrm{~B}$ ). This last fact combined with (3.2) shows that $X$ is bounded with respect to $\|\cdot\|_{1}$.

Now suppose that $\left\{f_{n}\right\}$ is a sequence in $X \cap A^{-1}[0, b]$ which converges weakly to a cycle $f \in \Sigma^{1}\left(\lambda ; \mathbf{R}^{N}\right)$. From general principles, $\left\{\left\|f_{n}\right\|_{1}\right\}$ is bounded, and $\left\|f_{n}\right\|_{0} \rightarrow\|f\|_{0}$ since weak $\Sigma^{1}$ convergence implies $C^{0}$ convergence. From (1.3) it follows that $\left\{\mathrm{J}\left(f_{n}\right)\right\}$ is bounded, and from (3.3) it follows that $\left\{\left|E\left(f_{n}\right)\right|\right\}$ is bounded. Hence Lemma $G$ guarantees that the $f_{n}$ are bounded away from $\mathrm{S}$, so that $f$ is homotopic to the $f_{n}$ in $\mathrm{R}^{N}-\mathrm{S}$, i.e., $f \in X$. To complete the proof we have to show that $f \in A^{-1}[0, b]$. We have $E\left(f_{n}\right) \rightarrow E(f)$ since weak convergence in $\Sigma^{1}$ implies $C^{0}$ convergence. Also, from general principles, we have $\|f\|_{1} \leqslant \varlimsup \lim \left\|f_{n}\right\|_{1}$, so that $J(f) \leqslant \varlimsup J\left(f_{n}\right)$. It follows that

$$
A(f)=J(f)-E(f) \leqslant \varlimsup
$$

and the desired result follows.

This completes the proof that $A \mid X$ attains its infimum. The proof of the corresponding statement for $A \mid \Omega^{1}\left(T ; p, q ; \mathbf{R}^{N}-\mathrm{S}\right)$ is similar.

3C. The proof that $J$ attains minimum values on the components of $\Sigma_{c}^{1}$ (and $\Omega_{c}^{1}$ ) described in the statement of Theorem 1.2 is also similar to the proof above. One uses Proposition 3.1 with $F=J$ and $X=$ a component in $\Sigma_{c}^{1}\left(\Omega_{c}^{1}\right)$ satisfying the given conditions. Again, the exclusion of certain homotopy classes is required to insure the nonvanishing of the Lagrange multiplier $\mu$.

4. Proof of Theorem 1.3. The Jacobi metric.

4A. Condition (SF)*. The proof of the theorem is especially easy if the SF condition is replaced with a slightly stronger condition ((SF)*), which has an interesting geometrical interpretation.

There exists a $C^{2}$ function $U$ on $\mathbf{R}^{N}-\mathbf{S}$ such that

$(\mathrm{SF})^{*}$

(i)* $U$ is proper on $\mathbf{R}^{N}-\mathrm{S}$,

(ii)* $-V \geqslant|\nabla U|^{2}$ on $\mathbf{R}^{N}-\mathrm{S}$.

Recall that a function $U$ is proper if and only if $U^{-1}[a, b]$ is compact for every compact interval $[a, b]$. Hence (i)* implies (i) of condition (SF), so that condition (SF)* implies condition (SF). In some cases the difference between these two conditions is only apparent. For example, if $\mathbf{S}$ is compact and $\boldsymbol{U}$ satisfies (i) and (ii) of the SF condition, then one can always obtain a comparison function satisfying (i)* and (ii)* by modifying the behavior of $U$ in the neighborhood of $\infty$. 
4B. The Jacobi metric. It turns out that condition (SF)* is precisely the condition which guarantees the completeness of $\mathrm{R}^{N}-\mathrm{S}$ (in the riemannian sense) with respect to the "Jacobi metric" $\hat{g}_{i j}$ where

$$
\hat{g}_{i j}=(h-V) \delta_{i j}
$$

Once this is established, the theorem will follow directly from the standard results of riemannian geometry.

To be more precise, let $\mathbf{M}$ be a riemannian manifold endowed with metric tensor $g=\left(g_{i j}\right)$, and let $V$ be a potential function on $M$. By definition, the Jacobi metric $\hat{g}$ corresponding to a fixed number $h$ is defined by $\hat{g}_{i j}=$ $(h-V) g_{i j}$. In our case $\mathbf{M}=\mathbf{R}^{N}-\mathbf{S}$ and $g_{i j}=\delta_{i j}$. According to a well-known variational principle of Jacobi, the nonconstant geodesics with respect to $\hat{g}$ are reparametrized solutions to $(1.1)$ with total energy $h$. (See e.g. [11, p. 125] for a rigorous statement and proof of this principle.)

Now, besides being extremals for the arc length functional $f \rightarrow \int|D f(t)| d t$, geodesics are also extremals for the "energy" functional $f \rightarrow \int|D f(t)|^{2} d t$. (See e.g. [13, p. 70] and also [11].) Hence in order to prove Theorem 1.3 it will suffice to show that the energy integral $T$ attains minimum values, where

$$
T=\int(h-V)|\dot{x}(t)|^{2} d t .
$$

But it is known that the energy functional attains minimum values, provided that the riemannian manifold is complete, i.e., the theorem will be proved once it is established that the metric $\hat{g}_{i j}$ defined by (4.1) is complete.

4C. A completeness criterion. The equivalence of condition (SF)* with the completeness of the metric $\hat{g}$ is an immediate consequence of the following proposition, whose proof is found in [10].

Proposition 4.1. In order that a smooth riemannian $\mathbf{M}$ be complete it is necessary and sufficient that $\mathbf{M}$ support a proper function $U$ whose gradient is bounded in norm.

Suppose now we are given condition (SF)*. Then raising tensor indices in the customary fashion, and setting $|\cdot|_{\hat{g}}=$ norm with respect to $\hat{g}$ and $\nabla_{\hat{g}}=$ gradient with respect to $\hat{g}$, we get

$$
\hat{g}^{i j}=(h-V)^{-1} \delta_{i j}
$$

and therefore

$$
\left|\nabla_{\hat{g}} U\right|_{\hat{g}}^{2}=\hat{g}^{i j} U_{, i} U_{, j}=(h-V)^{-1}|\nabla U|^{2}
$$

$\leqslant$ constant from (ii)*. 
4D. Proof of Theorem 1.3 under hypothesis (SF). We shall now briefly describe a proof which is valid when the more general SF condition is assumed. The proof follows the proof of Theorem 1.1(b) given in §3B and makes use of the lower semicontinuity properties of the functional $T$. In the proof one uses the following lemma, which has the same function as Lemma $G$ in the proof of Theorem 1.1.

LEMMA G*. Assume condition (SF), and in addition that $V<0$ and $h>0$. Let $T$ be the functional defined by (4.2). Then for any number $b$ the set of all paths $f$ in $\Omega^{1}\left(T ; p, q ; \mathbf{R}^{N}-\mathrm{S}\right)$ for which $T(f) \leqslant b$ is bounded away from $S$.

Corollary 4.2. Assuming the hypotheses of Lemma $\mathrm{G}^{*}$, then $T \mid \Omega^{1}\left(T ; p, q ; \mathbf{R}^{N}-\mathbf{S}\right)$ attains minimum values on components.

REMARK 4.1. The Euler-Lagrange equations for (4.2) are

$$
2(h-V) \ddot{x}-2\langle\dot{x}, \nabla V\rangle \dot{x}+|\dot{x}|^{2} \nabla V=0 .
$$

One cannot assert that the solutions to (1.1) which we obtain by minimizing $T$ on components of $\Omega^{1}\left(T ; p, q ; \mathbf{R}^{N}-\mathrm{S}\right)$ join $p$ to $q$ in time $T$ because of the reparametrization involved in going from (4.3) to (1.1).

To prove the lemma we first note that $2 h J(f) \leqslant T(f)$. Then using the notation of $\S 2 \mathrm{~A}$ we have

$$
\dot{\sigma}^{2}=|\dot{x}|^{2}+\langle\dot{x}, \nabla U\rangle^{2} \leqslant|\dot{x}|^{2}\left(1+|\nabla U|^{2}\right) \leqslant|\dot{x}|^{2}(1-V),
$$

so that

$$
\int_{0}^{T} \dot{\sigma}^{2} d t \leqslant(1+|1-1 / h|) T(f) .
$$

Hence $T(f) \leqslant$ constant implies $\sigma(\tilde{f}) \leqslant$ constant, and the proof proceeds very much as before.

4E. A method applicable to weak-force systems and the case $h<0$. We conclude this section by mentioning a method for constructing domains $D$ with the property that any two points in $\mathbf{D}$ can be joined by a smooth path which minimizes $T$. This method is applicable to weak-force systems as well as the case $h<0$, and is based on the following theorem whose proof is given in [12].

THEOREM. Let D be a connected open subset of a (not necessarily complete) riemannian manifold $\mathbf{M}$. Then in order that every two points in $\mathbf{D}$ be joined by a smooth geodesic minimizing arc length, it is sufficient that D support a smooth function $F$ which is proper, positive and convex.

In applying this theorem to the dynamical case one constructs functions $F$ 
which are convex with respect to the Jacobi metric. Also, the domains D need not have compact closure or be homeomorphic to euclidean balls; see [12] for details and examples.

5. Function-analytic preliminaries-the periodic case. In this section we shall assemble some well-known facts about Sobolev spaces of periodic $\mathbf{R}^{\boldsymbol{N}}$-valued functions. Since a space of $\lambda$-periodic $\mathbf{R}^{N}$-valued functions $(\lambda$ fixed) can be identified with a space of $\mathbf{R}^{N}$-valued maps on the circle $S^{\mathbf{1}}$, the theory presented here is merely the simplest example of a more general theory of Sobolev spaces of vector-valued functions defined on closed manifolds. For references see, e.g., [15], [17], [21].

5A. Notation and definitions. The standard inner product and corresponding norm of $\mathbf{R}^{N}$ will be denoted by $\langle$,$\rangle and |\cdot|$. Fix a number $\lambda>0$, and let $\Sigma^{\infty}=\Sigma^{\infty}\left(\lambda ; \mathbf{R}^{N}\right)$ represent the space of all $C^{\infty} \lambda$-periodic maps from $\mathbf{R}$ to $\mathbf{R}^{N}$ (so that $\Sigma^{\infty} \approx C^{\infty}\left(S^{1}, \mathbf{R}^{N}\right.$ ). We write $D$ for $d / d t$, and for $f, g \in \Sigma^{1}$ we set

$$
\begin{aligned}
& (f, g)_{0}=\int_{0}^{\lambda}\langle f(t), g(t)\rangle d t, \quad\|f\|_{0}^{2}=(f, f)_{0}, \\
& (f, g)_{1}=(f, g)_{0}+(D f, D g)_{0}, \quad\|f\|_{1}^{2}=(f, f)_{1}, \\
& \|f\|_{-1}=\sup \left\{(f, g)_{0},\|g\|_{1}=1\right\} .
\end{aligned}
$$

The second term of the right-hand side of (5.2) can be integrated by parts to obtain

$$
(f, g)_{1}=\left(\left(I-D^{2}\right) f, g\right)_{0} .
$$

Note that $I-D^{2}$ is an elliptic operator with trivial kernel.

The Sobolev spaces $\Sigma^{i}=\Sigma^{i}\left(\lambda ; \mathbf{R}^{N}\right), i=-1,0,1$, are defined to be the completions of $\Sigma^{\infty}$ with respect to $\|\cdot\|_{i}$. We recall the following well-known facts:

(a) (Rellich's theorem). $\Sigma^{1} \subset \Sigma^{0} \subset \Sigma^{-1}$, and the injections $\Sigma^{1} \rightarrow \Sigma^{0}$, $\Sigma^{0} \rightarrow \Sigma^{-1}$ are compact (completely continuous).

(b) (Sobolev's theorem). Weak $\Sigma^{1}$-convergence implies $C^{0}$ convergence. Hence the functions belonging to $\Sigma^{\mathbf{1}}$ are continuous (in fact, absolutely continuous).

(c) $(,)_{0}$ is a continuous bilinear function on $\Sigma^{\mathbf{1}}$. Hence there exists a compact operator $G$ on $\Sigma^{1}$ such that

$$
(f, g)_{0}=(G f, g)_{1}, \quad f, g \in \Sigma^{1} .
$$

The kernel of $G$ is trivial, so that the eigenvectors of $G$ constitute an orthonormal basis for $\Sigma^{1}$. (See, e.g., Riesz and Sz.-Nagy [19, p. 234].) 
(d) $\left(I-D^{2}\right)$ is an isomorphism from $\Sigma^{1}$ onto $\Sigma^{-1}$, and $G$ extends to an inverse isomorphism from $\Sigma^{-1}$ to $\Sigma^{1}$. In other words, $G$. is the Green's operator associated with the elliptic operator $\left(I-D^{2}\right)$ and we can write

$$
\left(I-D^{2}\right) G=G\left(I-D^{2}\right)=I .
$$

(e) $\Sigma^{-1}$ is in fact a hilbert space with inner product

$$
(f, g)_{-1}=(G f, g)_{0} \text {. }
$$

Also, $(,)_{0}$ considered as a bilinear form on $C^{\infty}$ extends to a continuous bilinear function on $\Sigma^{1} \times \Sigma^{-1}$, and $\Sigma^{1}$ is dual to $\Sigma^{1}$ through $(,)_{0}$, i.e., for every continuous linear functional $L$ on $\Sigma^{1}$ there exists a $g \in \Sigma^{-1}$ such that $L=$ $(\cdot, g)_{0}$. (It is thus apparent that $\Sigma^{-1}$ is a space of Schwartz distributions (on $\left.S^{1}\right)$ which are of the same order as the (vector) Dirac $\delta$-functions.)

5B. The gradients $\nabla^{0}$ and $\nabla^{1}$. Proof of Lemma $A_{1}$. Let $F$ be a realvalued function on $\Sigma^{1}$ of class $C^{1}$. The $\Sigma^{1}$-gradient of $F$ at $f \in \Sigma^{1}$ is defined by the relation

$$
d F_{f}(v)=\left.\frac{d}{d \epsilon} F(f+\epsilon v)\right|_{\epsilon=0}=\left(\nabla^{1} F(f), v\right)_{1}
$$

which must hold for every $v$ in $\Sigma^{1}$, so that from (e) we must have

$$
d F_{f}(v)=\left(\nabla^{0} F(f), v\right)_{0}
$$

for some element $\nabla^{0} F(f)$ of $\Sigma^{-1}$. From (5.5) it follows that

$$
\nabla^{1} F=G \nabla^{0} F \text {. }
$$

Now suppose that

$$
F(f)=\int_{0}^{\lambda} Q(x(t), \dot{x}(t)) d t
$$

where $Q=Q(x, \dot{x})$ is a $C^{2}$ function of $2 N$ real variables, and $x=x(t)$ is the representation of $f \in \Sigma^{1}$ in euclidean coordinates. Then, formally at least, we have

$$
d F_{f}(v)=\left(\frac{\partial Q}{\partial x}, v\right)_{0}+\left(\frac{\partial Q}{\partial \dot{x}}, \dot{v}\right)_{0}=\left(\frac{\partial Q}{\partial x}-\left(\frac{d}{d t} \frac{\partial Q}{\partial \dot{x}}\right), v\right)_{0},
$$

i.e.

$$
\nabla^{0} F(f)=\frac{\partial Q}{\partial x}-\frac{d}{d t} \frac{\partial Q}{\partial \dot{x}} .
$$

In other words, the distribution $\nabla^{0} F(f)$ can be identified with the "first variation" of $F$ at $f$. 
In particular, we have (from (1.3) and (5.6))

$$
\begin{aligned}
& \nabla^{0} J(f)=-D^{2} f, \quad \nabla^{1} J(f)=-G D^{2} f=f-G f, \\
& \nabla^{0} E(f)=\nabla V(f), \quad \nabla^{1} E(f)=G \nabla V(f), \\
& \nabla^{0} A(f)=-D^{2} f-\nabla V(f), \quad \nabla^{1} A(f)=f-G f-G \nabla V(f) .
\end{aligned}
$$

In order that these last relations (and their derivations) make sense it is necessary that the operators $D$ and $D^{2}$ be interpreted in the distribution sense, i.e., we consider $D$ to be a continuous map from $\Sigma^{i}$ to $\Sigma^{i-1}(i=0,1)$ and $D^{2}$ to be a continuous map from $\Sigma^{1}$ to $\Sigma^{-1}$. For example, for $u \in \Sigma^{0}$ and $v \in \Sigma^{1}$ we can write

$$
(u, D v)_{0}=-(D u, v)_{0} .
$$

One way of justifying this relation is to replace the functions $u, v$ with sequences of $C^{\infty}$ functions which converge to $u$ and $v$ in the $\Sigma^{0}, \Sigma^{1}$ topologies, and then pass to the limit on each side (cf. (e)).

The proof of Lemma $A_{1}$ is now an immediate consequence of (5.11).

6. The Palais-Smale condition and the behavior of families of periodic orbits.

6A. Let $M$ be a smooth riemannian manifold which is possibly infinite dimensional. Recall that a $C^{0}$ function $F$ on $\mathbf{M}$ which is bounded below is said to satisfy the Palais-Smale (P-S) condition (often referred to as "Condition C" in the literature) if every subset of $\mathbf{M}$ on which $F$ is bounded and $|\nabla F|$ is not bounded away from zero contains a critical point of $F$ in its closure. It is known that the Morse and Lusternik-Schnirelmann critical point theories can be applied to functions which satisfy the P-S condition provided that $\mathbf{M}$ is complete; in particular, such functions attain minimum values on each component of $\mathbf{M}$ ([14], [16], [19]). If $\mathbf{M}$ is not complete, the following condition is required for the theory to hold [16, p. 208].

For every real number $a, f^{-1}(-\infty, a]$ is complete.

Of course, the Morse and Lusternik-Schnirelmann theories provide for the existence of critical points of a more general type than local minima, and in our particular case the application of such results would require topological data on the loop spaces of our configuration spaces $\mathbf{R}^{N}-\mathbf{S}$. We shall not pursue this line of inquiry in this paper, but shall be content to prove the following theorems.

THEOREM 6.1. Assume condition (SF) and in addition that $V<0$, and let $\Gamma$ be any component of $\Sigma^{\mathbf{1}}\left(\lambda ; \mathbf{R}^{N}-\mathrm{S}\right)$ which consists of cycles tied to $\mathbf{S}$. Then AI $\Gamma$ satisfies the P-S condition. 
THEOREM 6.2. Assume condition (SF) and let $\Gamma_{c}$ be any component of $\Sigma_{c}^{1}\left(\lambda ; \mathbf{R}^{N}-\mathrm{S}\right)$ which consists of cycles tied to $\mathbf{S}$. Then $J \mid \Gamma_{c}$ satisfies the P-S condition.

Proofs. The spaces $\Sigma^{1}\left(\lambda ; \mathbf{R}^{N}-S\right)$ and $\Sigma_{c}^{1}\left(\lambda ; \mathbf{R}^{N}-S\right)$ are incomplete, but Lemma $G$ renders this lack of completeness ignorable, i.e., from Lemma $G$ it follows that the SF condition implies property $(*)$ for the functions $F=A \mid \Gamma$ and $F=J \mid \Gamma_{c}$. Indeed, in $\S 3 \mathrm{~B}, \mathrm{C}$ it was shown that $\Gamma \cap \mathrm{A}^{-1}(-\infty, b]$ and $\Gamma_{c} \cap J^{-1}(-\infty, b]$ are weakly compact (and therefore closed) subsets of $\Sigma^{1}\left(\lambda ; \mathbf{R}^{N}\right)$ and $\Sigma_{c}^{1}\left(\lambda ; \mathbf{R}^{N}\right)$.

Proceeding now with the proof of Theorem 6.1, suppose we are given a sequence $\left\{f_{n}\right\}$ of cycles in $\Sigma^{1}\left(\lambda ; \mathbf{R}^{N}-\mathrm{S}\right)$ for which $A\left(f_{n}\right) \leqslant b$ and $\nabla^{1} A\left(f_{n}\right)$ $\rightarrow 0$ in the strong $\Sigma^{1}$ topology. Using (5.11) we have

$$
f_{n}-G f_{n}-G \nabla V\left(f_{n}\right) \stackrel{\text { strong, } \Sigma^{1}}{\longrightarrow} 0 \text {. }
$$

But from the weak compactness of $A^{-1}(-\infty, b]$ it follows that by passing to a subsequence we can assume that $f_{n} \rightarrow f$ weakly in $\Sigma^{1}$ for some $f$ in $\Sigma^{1}$. And from the compactness property of $G$, the weak convergence of $f_{n}$ implies the strong convergence of $G f_{n}$ and also $G \nabla V\left(f_{n}\right)$. Hence from (6.1) it follows that $f_{n}$ converges strongly.

The proof of Theorem 6.2 is similar, except that the relation $\nabla^{1} \mathrm{~A}\left(f_{n}\right) \rightarrow 0$ is replaced with

$$
\nabla^{1} J\left(f_{n}\right)-\mu\left(f_{n}\right) \nabla^{1} E\left(f_{n}\right) \stackrel{\text { strong, } \Sigma^{1}}{\longrightarrow} 0
$$

where

$$
\mu_{n}=\frac{\left(\nabla^{1} J\left(f_{n}\right), \nabla^{1} E\left(f_{n}\right)\right)_{1}}{\left\|\nabla E\left(f_{n}\right)\right\|_{1}^{2}}
$$

i.e., (6.1) is replaced with

$$
f_{n}-G f_{n}=\mu\left(f_{n}\right) G \nabla V\left(f_{n}\right) \stackrel{\text { strong, } \Sigma^{1}}{\longrightarrow} 0 .
$$

By passing to a subsequence one can suppose that $\mu\left(f_{n}\right) \rightarrow$ constant, and the proof proceeds as before.

6B. The failure of the Palais-Smale condition for the gravitational case and the behavior of families of periodic orbits. As is well known, the Kepler problem, i.e., problem of two bodies moving under the influence of their mutual gravitation, is equivalent to the problem of one body moving in a plane around a fixed center of attraction according to the equation $\ddot{x}=-x /|x|^{3}$ (cf. Example 1.2).

Proposition 6.3. For the Kepler problem the functionals 


$$
A=A \mid \Sigma^{1}\left(\lambda ; R^{2}-(0,0)\right) \text { and } J \mid \Sigma_{c}^{1}\left(\lambda ; R^{2}-(0,0)\right)
$$

do not satisfy the P-S condition.

Proof. In the Kepler problem a trajectory is periodic if and only if it has total energy $h<0$, and orbits with equal periods have equal total energy $h$, and also equal averaged kinetic energy $J$ and averaged potential $E$. Let $\left\{f_{n}\right\}$ be a sequence of $\lambda$-periodic trajectories ( $\lambda$ fixed) whose perihelions converge to the origin as $n \rightarrow \infty$. Then by perturbing each $f_{n}$ slightly in a neighborhood of its aperihelion we obtain a sequence $\left\{f_{n}^{\prime}\right\}$ for which, say, $\left|A\left(f_{n}^{\prime}\right)\right|$ is bounded and $\nabla^{1} A\left(f_{n}^{\prime}\right) \rightarrow 0$, but for which the $f_{n}^{\prime}$ do not converge to a cycle in $\mathbf{R}^{2}-(0,0)$.

More generally, for a given hamiltonian system, suppose there exists a submanifold $\mathrm{M}$ of phase space consisting entirely of periodic orbits. Let $H, P, J$, and $E$ denote the hamiltonian, period, and averaged kinetic and potential energies, respectively, expressed as functions of $\mathbf{M}$, e.g., for $w \in \mathbf{M}$,

$$
E(w)=\frac{1}{P(w)} \int_{0}^{P(w)} V\left(\varphi_{t} w\right) d t
$$

where $\varphi_{t}$ is the hamiltonian flow. Suppose also that these functions are all of class $C^{1}$; then it is known that [6]

$$
0=d P \wedge d H=d P \wedge d J=d P \wedge d E=d H \wedge d J=d H \wedge d E=d J \wedge d E .
$$

Hence if $\lambda$ is not a critical value of $P$, then $H, J$ and $E$ are constant on components of $P^{-1}\{\lambda\}$. Combining this remark with Lemma G, we obtain the following theorem.

THEOREM 6.4. Suppose we are given a SF system in which a family $\mathbf{F}$ of $\lambda$-periodic trajectories ( $\lambda$ fixed) can be embedded as a connected subset of a smooth submanifold $\mathbf{M}$ of phase space consisting of periodic trajectories (of differing periods). Suppose also that $P, J$ and $E$ are of class $C^{1}$ on $\mathrm{M}$ and that $\lambda$ is not a critical value of $\boldsymbol{P}$ (restricted to $\mathbf{M}$ ). Then the family $F$ of $\lambda$-periodic trajectories is bounded away from $\mathbf{S}$.

The condition that $\lambda$ be a noncritical value can be replaced with the condition that $V<0$.

THEOREM 6.5. Assume condition SF and in addition that $V<0$. Let $\mathrm{F}$ be a family of $\lambda$-periodic trajectories ( $\lambda$ fixed) which can be embedded as a pathconnected subset of a manifold $\mathrm{M}$ of periodic trajectories on which $P, J$, and $E$ are of class $C^{1}$. Then $\mathbf{F}$ is bounded away from $\mathbf{S}$.

Proof. In $[6$, p. 113] it is shown that $-d P / P=d(J-E) / 2 J$. Hence $J-E=$ constant on $\mathrm{F}$, and since $E<0$ it follows that $J$ and $E$ are bounded on $\mathbf{F}$. 


\section{The nonperiodic case.}

7A. Preliminary remarks. The condition that a curve $f: x=x(t)$ be a critical point for the functional

$$
A=A \mid \Omega^{1}\left(T ; p, q ; \mathbf{R}^{N}-\mathbf{S}\right)
$$

is given by

$$
-d A_{f}(v)=(\ddot{x}+\nabla V(x), v)_{0}=0
$$

where this relation is required to hold for all smooth "test functions" $v=v(t)$ which vanish at $t=0$ and $t=T$, and the derivative $\ddot{x}$ is interpreted in a distribution sense. If $f$ were known to be of class $C^{2}$, so that. $\ddot{x}+\nabla V(x)$ is of class $C^{0}$, one could conclude that $\ddot{x}+\nabla V(x)=0$. In the absence of such knowledge, all one might conclude from (7.1) is that $\ddot{x}+\nabla V(x)$ is a distribution which consists of a linear combination of (vector) Dirac $\delta$-functions centered at $t=0$ and $t=T$. In other words, in order to prove Lemma $A_{2}(\S 1 D)$ and extend the results of the last two sections to the nonperiodic case of functionals defined on $\Omega^{1}\left(T ; p, q ; \mathbf{R}^{N}-\mathrm{S}\right)$, we have to establish some regularity results concerning the smoothness of distributions satisfying certain conditions. For example, it turns out that the Dirac functions which are centered at the endpoints correspond to $C^{\infty}$ functions, namely, exponentials.

7B. The Sobolev spaces $\Omega_{e}^{i}, \Omega_{p, q}^{1}, \Omega_{*}^{1}$. We now introduce certain spaces and operators in parallel to the development given in $\S 5 \mathrm{~A}$. On the space $C^{\infty}\left([0, T], \mathbf{R}^{N}\right)$ we define the inner products $(,)_{i}(i=0,1)$, and norms $\|\cdot\|_{i}$ $(i=-1,0,+1)$, exactly as in formulae (5.1), (5.2) and (5.3), and we define $\Omega_{e}^{i}=\Omega_{e}^{i}\left(T, \mathbf{R}^{N}\right)$ to be the completions of $C^{\infty}\left([0, T], \mathbf{R}^{N}\right)$ with respect to the corresponding norm. Note that (5.4) is not valid in the present case, since we no longer have integration by parts, i.e., for $f, g$ in $\Omega_{e}^{1}$ we can only write $(D f, g)_{0}=-(f, D g)_{0}$ if either $f$ or $g$ vanishes at the endpoints.

The theorems of Rellich and Sobolev remain valid, and one can define a compact selfadjoint operator $G$ by (5.5), so that $\Omega_{e}^{-1}$ becomes a hilbert space with inner product defined by (5.7). The relation $G\left(I-D^{2}\right)=I$ is no longer valid; indeed, the operator $\left(I-D^{2}\right)$ now has a nontrivial kernel. However, it will be shown that $G$ is a right-inverse to $\left(I-D^{2}\right)$, so that $\left(I-D^{2}\right)$ is a surjective map from $\Omega_{e}^{1}$ to $\Omega_{e}^{-1}$.

For the remainder of this section we set

$$
\Omega_{p, q}^{1}=\Omega^{1}\left(T ; p, q ; \mathbf{R}^{N}-\mathrm{S}\right),
$$

which we recall (§1B) is the set of all $f$ in $\Omega_{e}^{1}$ with values in $R^{N}-S$ for which $f(0)=p, f(T)=q$, and we let $\Omega_{*}^{1}$ represent the linear submanifold of $\Omega_{e}^{1}$ consisting of all $f$ in $\Omega_{e}^{1}$ for which $f(0)=f(T)=0$. 
The space $\Omega_{*}^{1}$ can be identified with the tangent space of $\Omega_{p, q}^{1}$ at any point, and we conclude this paragraph by computing the gradients of $J, E$ and $A$ (restricted to $\Omega_{p, q}^{1}$ ).

Let $P$ be the projection of $\Omega_{e}^{1}$ onto $\Omega_{*}^{1}$ which is orthogonal with respect to the inner product $(,)_{1}$. Then the formulae (5.11) of $\S 5 \mathrm{~B}$ are no longer valid, but we have

$$
\begin{aligned}
& \nabla^{1} J(f)=-P G D^{2} f, \quad \nabla^{1} E(f)=P G \nabla V(f), \\
& \nabla^{1} A(f)=-P G\left(D^{2} f+\nabla V(f)\right) .
\end{aligned}
$$

It will suffice to prove the first relation. By definition, $\nabla^{1} J$ is the element of the tangent space $\left(\Omega_{*}^{1}\right)$ of $\Omega_{p, q}^{1}$ which satisfies $d J_{f}(v)=\left(\nabla^{1} J(f), v\right)_{1}$ for all $v$ in $\Omega_{*}^{1}$. But $d J_{f}(v)=(D f, D v)_{0}$, and since $v \in \Omega_{*}^{1}$, we can write $d J_{f}(v)=$ - $\left(D^{2} f, v\right)_{0}$ where the meaning of the right-hand side in the sense of distributions is again indicated by the discussion following (5.12). Continuing, we have

$$
d J_{f}(v)=-\left(D^{2} f, v\right)_{0}=-\left(G D^{2} f, v\right)_{1}=-\left(G D^{2} f, P v\right)_{1}=-\left(P G D^{2} f, v\right)_{1} .
$$

7C. Fundamental results on regularity. Proof of Lemma $\mathrm{A}_{2}$. We shall now present some lemmas which are involved in proofs of theorems which are the analogues of the results of $\S 6$ for the nonperiodic case. The presentation given here has some features in common with Palais' proof in [14] that the energy integral satisfies the P.S condition, but unfortunately we were not able to adapt his proof (the case $V \equiv 0$ ) for our purposes.

Lemma 7.1. $\left(I-D^{2}\right) G=I$, where $G$ and $\left(I-D^{2}\right)$ are considered as maps from $\Omega_{e}^{-1}$ to $\Omega_{e}^{1}$ and $\Omega_{e}^{1}$ to $\Omega_{e}^{-1}$ respectively.

LEMma 7.2. The orthogonal complement $\left(\Omega_{*}^{1}\right)^{\perp}$ of $\Omega_{*}^{1}$ in $\Omega_{e}^{1}$, i.e., the range of $(I-P)$, has dimension $2 N$ and consists entirely of $C^{\infty}$ functions. Specifically, $\left(\Omega_{*}^{1}\right)^{\perp}$ consists of maps $f$ of the form

$$
f(t)=a e^{t}+b e^{-t}, \quad a, b \in \mathbf{R}^{N} .
$$

Corollary. $f \in \Omega_{e}^{1}$ and $P f=0$ implies $f \in C^{\infty}$.

Lemma 7.3. $f \in \Omega_{e}^{-1}$ and $P G f=0$ implies $f=0$.

REMARK. Lemma $A_{2}$ is an immediate consequence of Lemma 7.3 and the relations (7.2).

LEMMA 7.4. Let $f, f_{n}$ be elements of $\Omega_{e}^{1}$ such that

$$
f_{n} \stackrel{\text { weak, } \Omega_{e}^{1}}{\longrightarrow} f \text { and } D^{2} f_{n} \stackrel{\text { strong, } \Omega_{e}^{-1}}{\longrightarrow} D^{2} f
$$

Then 


$$
f_{n} \stackrel{\text { strong, } \Omega_{e}^{1}}{\longrightarrow} f .
$$

Proofs. [7.1]. It suffices to prove that $\left(I-D^{2}\right) G f=f$ for all $f$ of class $C^{\infty}$ since $C^{\infty}$ is dense in $\Omega_{e}^{1}$ and $\left(I-D^{2}\right) G$ is a continuous map from $\Omega_{e}^{1}$ to $\Omega_{e}^{1}$. For any $v$ in $\Omega_{*}^{1}$ we can integrate by parts to obtain

$$
\left(\left(I-D^{2}\right) G f, v\right)_{0}=(G f, v)_{0}+(D G f, D v)_{0}=(G f, v)_{1}=(f, v)_{0},
$$

and since $f$ and $\left(I-D^{2}\right) G f$ are of class $C^{0}$ the result follows.

[7.2]. A direct calculation shows that every $f$ of the form (7.3) belongs to $\left(\Omega_{*}^{1}\right)^{\perp}$. In fact, for $u \in \Omega_{e}^{1}$ we have

$$
\left(u, a e^{t}+b e^{-t}\right)_{1}=\left.\left\langle u(t), a e^{t}-b e^{-t}\right\rangle\right|_{t=0} ^{t=T} .
$$

To conclude the proof, it suffices to show that $\operatorname{dim}\left(\Omega_{*}^{1}\right)^{\perp} \leqslant 2 N$. Now, by the Sobolev theorems, the map that sends every $f \in \Omega_{e}^{1}$ into its $i$ th component evaluated at $t=0$ or $t=T$ is a continuous linear functional on $\Omega_{e}^{1}$, and therefore, by the Riesz representation theorem, there exist $2 N$ elements $\alpha_{1}, \cdots, \alpha_{2 N}$ of $\Omega_{e}^{1}$ such that $\Omega_{*}^{1}$ consists precisely of those $f$ in $\Omega_{e}^{1}$ for which

$$
\left(f, \alpha_{1}\right)_{1}=\left(f, \alpha_{2}\right)_{1}=\cdots=\left(f, \alpha_{2 N}\right)_{1}=0
$$

The result follows.

[7.3]. From Lemma 7.2 we have

$$
\left(I-D^{2}\right)(I-P)=0
$$

and $G f=P G f+(I-P) G f=(I-P) G f$ by hypothesis. Now apply $\left(I-D^{2}\right)$ to both sides and apply Lemma 7.1 and equation (7.5).

[7.4]. Without loss of generality we can assume $f=0$, and the problem is to show that $f_{n} \rightarrow 0$ strongly in $\Omega_{e}^{1}$. But $f_{n} \rightarrow 0$ weakly and from Lemma 7.2 the operator $(I-P)$ is compact, so that $(I-P) f_{n} \rightarrow 0$ strongly in $\Omega_{e}^{1}$. Therefore, it suffices to show that $P f_{n} \rightarrow 0$ strongly in $\Omega_{e}^{1}$. Again, since $(I-P) f_{n} \rightarrow 0$ strongly in $\Omega_{e}^{1}$, it follows that $D^{2}(I-P) f_{n} \rightarrow 0$ strongly in $\Omega_{e}^{-1}$; and since $D^{2} f_{n} \rightarrow 0$ strongly in $\Omega_{e}^{-1}$ by hypothesis, it follows that $D^{2} P f_{n} \rightarrow 0$ strongly in $\Omega_{e}^{-1}$.

Since $P f_{n} \in \Omega_{*}^{1}$, we can integrate by parts to obtain

$$
\left\|D P f_{n}\right\|_{0}^{2}=-\left(D^{2} P f_{n}, P f_{n}\right)_{0} \leqslant\left\|D^{2} P f_{n}\right\|_{-1} \cdot\left\|P f_{n}\right\|_{1} \rightarrow 0
$$

Hence

$$
\left\|P f_{n}\right\|_{1}^{2}=\left\|D P f_{n}\right\|_{0}^{2}+\left\|P f_{n}\right\|_{0}^{2} \rightarrow 0
$$

and the lemma is proved. 
7D. The Palais-Smale condition. As an illustration of how these lemmas can be used to establish that the results of $\S 6 \mathrm{~A}$ have analogues inthe nonperiodic case, we prove the following theorem.

THEOREM 7.1. Assume condition (SF) and in addition that $V<0$. Then $A=A \mid \Omega_{p, q}^{1}$ satisfies the P-S condition.

Proof. As in the periodic case, we obtain a sequence $\left\{f_{n}\right\}$ of maps in $\Omega_{p, q}^{1}$ which are bounded away from $S$, converge in the weak $\Omega_{e}^{1}$ topology, and satisfy

$$
\nabla^{1} A\left(f_{n}\right)=-P G\left(D^{2} f_{n}+\nabla V\left(f_{n}\right)\right) \stackrel{\text { strong, } \Omega_{e}^{1}}{\longrightarrow} 0 .
$$

But from the continuity of $G D^{2}: \Omega_{e}^{1} \rightarrow \Omega_{e}^{1}$ and the compactness of $(I-P)$, $(I-P) G\left(D^{2} f_{n}+\nabla V\left(f_{n}\right)\right)$ also converges strongly in $\Omega_{e}^{1}$. Hence $G\left(D^{2} f_{n}+\nabla V\left(f_{n}\right)\right)$ converges strongly in $\Omega_{e}^{1}$, and using Lemma 7.1 , it follows that $D^{2} f_{n}+\nabla V\left(f_{n}\right)$ converges strongly in $\Omega_{e}^{-1}$. But from the Sobolev theorems $\nabla V\left(f_{n}\right)$ converges in the $C^{0}$ sense, and therefore strongly in $\Omega_{e}^{-1}$; hence $D^{2} f_{n}$ also converges strongly in $\Omega_{e}^{-1}$, and the desired result follows from Lemma 7.4.

\section{REFERENCES}

1. N. I. Ahiezer, The calculus of variations, GITTL, Moscow, 1955; English transl., Blaisdell, Waltham, Mass., 1962. MR 17, 861; 25 \#5414.

2. M. S. Berger, On periodic solutions of second order Hamiltonian systems. I, J. Math. Anal. Appl. 29 (1970), 512-522. MR 41 \#2121.

3. - Multiple solutions of non-linear operator equations arising from the calculus of variations, Proc. Sympos. Pure Math., vol. 18, part 1, Amer. Math. Soc., Providence, R. I., 1970, pp. 10-27. MR 42 \#5112.

4. - Periodic solutions of second order dynamical systems and isoperimetric variational problems, Amer. J. Math. 93 (1971), 1-10. MR 43 \#2588.

5. I. M. Gel' fand and S. V. Fomin, Calculus of variations, Fizmatgiz, Moscow, 1961; English transl., Prentice-Hall, Englewood Cliffs, N. J., 1963. MR 28 \#3352; \#3353.

6. W. B. Gordon, On the relation between period and energy in periodic dynamical systems, J. Math. Mech. 19 (1969/70), 111-114. MR 39 \#7236.

7. - A theorem on the existence of periodic solutions to Hamiltonian systems with convex potential, J. Differential Equations 10 (1971), 324-335. MR 45 \#1200.

8. - Periodic solutions to hamiltonian systems with infinitely deep potential wells, Ordinary Differential Equations, Academic Press, New York, 1972, pp. 399-403.

9. - Physical variational principles which satisfy the Palais-Smale condition, Bull. Amer. Math. Soc. 78 (1972), 712-716. MR 45 \#8080.

10. - An analytical criterion for the completeness of riemannian manifolds, Proc. Amer. Math. Soc. 37 (1973), 221-225; Corrections, ibid. 45 (1974), 130-131.

11. - On the equivalence of second order systems occuring in the calculus of variations, Arch. Rational Mech. Anal. 50 (1973), 118-126.

12. - On the existence of geodesics joining two given points, J. Differential Geometry 9 (1974), 443-450.

13. J. Milnor, Morse theory, Ann. of Math. Studies, no. 51, Princeton Univ. Press, Princeton, N. J., 1963. MR 29 \#634.

14. R. S. Palais, Morse theory on hilbert manifolds, Topology 2 (1963), 299-340. MR 28 \#1633. 
15. R. S. Palais, Foundations of global non-linear analysis, Benjamin, New York, 1968. MR 40 \#2130.

16. Critical point theory und the minimax principle, Proc. Sympos. Pure Math., vol. 15, Amer. Math. Soc., Providence, R. I., 1970, pp. 185-212. MR 41 \#9303.

17. R. S. Palais et al., Seminar on the A tiyah-Singer index theorem, Ann. of Math. Studies, no. 57, Princeton Univ. Press, Princeton, N. J., 1965. MR 33 \#6649.

18. R. S. Palais and S. Smale, A generalized Morse theory, Bull. Amer. Math. Soc. 70 (1964), 165-171.

19. F. Riesz and B. Sz.-Nagy, Leçons d'analyse fonctionnelle, 2ième éd., Akad. Kiadó, Budapest, 1953; English transl., Functional analysis, Ungar, New York, 1955. MR 15, 132 ; $17,175$.

20. J. T. Schwartz, Generalizing the Lusternik-Schnirelman theory of critical points, Comm. Pure Appl. Math. 17 (1964), 307-31 5. MR 29 \#4069.

21. F. W. Warner, Foundations of differentiable manifolds and Lie groups, Scott, Foresman, Glenview, III., 1971. MR 45 \#4312.

MATHEMATICS RESEARCH CENTER, NAVAL RESEARCH LABORATORY, WASHINGTON, D. C. 20375 